\title{
A Comparison of the Microwave Photoconductivity Decay and Open-Circuit Voltage Decay Lifetime Measurement Techniques for Lifetime-Enhanced 4H-SiC Epilayers
}

\author{
EDWARD VAN BRUNT, ${ }^{1,3}$ ANANT AGARWAL, ${ }^{2}$ AL BURK, ${ }^{1}$ LIN CHENG, ${ }^{1}$ \\ MICHAEL O'LOUGHLIN, ${ }^{1}$ JOHN PALMOUR, ${ }^{1}$ \\ and ALEXANDER SUVOROV ${ }^{1}$
}

1.-Cree, Inc., 4600 Silicon Drive, Durham, NC 27703, USA. 2.-U.S. Department of Energy, 1000 Independence Ave. SW, Washington, DC 20585, USA. 3.-e-mail: edward.vanbrunt@cree.com

\begin{abstract}
This work compares the optical microwave photoconductivity decay ( $\mu \mathrm{PCD})$ and electrical open-circuit voltage decay (OCVD) techniques for measuring the ambipolar carrier lifetime in $4 \mathrm{H}$-silicon carbide $(4 \mathrm{H}-\mathrm{SiC})$ epitaxial layers. Lifetime measurements were carried out by fabricating $\mathrm{P}^{+} /$intrinsic $/ \mathrm{N}^{+}(\mathrm{PiN})$ diodes on $100-\mu \mathrm{m}$-thick, $1 \times 10^{14} \mathrm{~cm}^{-3}$ to $4.5 \times 10^{14} \mathrm{~cm}^{-3}$ doped N-type 4 $\mathrm{H}$-SiC epilayers, and measuring the lifetime optically using $\mu \mathrm{PCD}$ prior to metallization, then electrically using OCVD after contact deposition. Both as-grown epilayers as well as epilayers with improved lifetime (via thermal oxidation) were measured using both techniques. The observed ambipolar lifetime was improved from $1.4 \mu \mathrm{s}$ on an unenhanced wafer to $4 \mu \mathrm{s}$ on a wafer enhanced through the oxidation process as measured by $\mu \mathrm{PCD}$. Little difference was observed between the $\mu \mathrm{PCD}$ and OCVD measurements on the unenhanced wafer; the ambipolar lifetime on the enhanced wafer measured by OCVD was approximately $5.5 \mu \mathrm{s}$, or $1.5 \mu \mathrm{s}$ higher than the $\mu$ PCD measurement. Continuous evaluation of the OCVD transient waveform was necessary due to the high lifetime in the enhanced wafer; shunt resistances included to discharge the $\mathrm{P}^{+} / \mathrm{N}$ junction capacitance were found to damp the OCVD response and yield low values for the measured lifetime. Simulation of the $\mu \mathrm{PCD}$ measurement including various surface recombination conditions yielded a good match to experimentally observed $\mu \mathrm{PCD}$ measurements for high values of the surface recombination velocity. The OCVD lifetime measurement technique is expected to yield measured lifetime values closer to the physical value due to its independence from surface conditions, provided that the experimental conditions are appropriately chosen.
\end{abstract}

Key words: Silicon carbide, minority carrier lifetime, lifetime measurement, lifetime enhancement

\section{INTRODUCTION}

Recent advances in $4 \mathrm{H}$-silicon carbide (4H-SiC) epitaxial growth have resulted in the availability of thick $(100 \mu \mathrm{m}+)$ material suitable for fabrication of bipolar devices such as insulated gate bipolar transistors (IGBTs), thyristors, and $\mathrm{P}^{+} /$intrinsic/ $\mathrm{N}^{+}(\mathrm{PiN})$

(Received August 8, 2013; accepted September 26, 2013; published online October 31, 2013) diodes that are free from bipolar drift. ${ }^{1,2}$ Recently, a great deal of research effort has been focused on improving the ambipolar carrier lifetime of $4 \mathrm{H}-\mathrm{SiC}$ epilayers, which has resulted in the development of techniques to improve the lifetime from typical as-grown lifetimes of about $1 \mu$ s to tens of microseconds. ${ }^{3,4}$ The ability to measure ambipolar carrier lifetimes close to the true material lifetime allows for better predictions of device performance, as well as enabling a more informed device design cycle by 
improving the accuracy of two dimensional (2D) numerical simulations. Ambipolar carrier lifetime is typically measured using either optical techniques [such as time-resolved photoluminescence (TRPL), transient free carrier absorption (TFCA), or microwave photoconductivity decay $\left.(\mu \mathrm{PCD})^{5}\right]$ on as-grown epilayers, or using electrical techniques [such as current recovery time (CRT) or open-circuit voltage decay $\left.(\mathrm{OCVD})^{6}\right]$ on fabricated devices. Each measurement technique is sensitive to different measurement artifacts. In general, optical techniques are sensitive to, and can be dominated by, surface recombination, whereas differences in experimental setup can cause discrepancies in electrical measurements.

There are few studies available that attempt to correlate electrical and optical measurements, and those that do exist have shown that optical measurements correlate poorly with electrical lifetime measurements. ${ }^{7}$ This work details the results of lifetime measurements conducted using both the OCVD and $\mu$ PCD techniques, and provides an explanation to reconcile the differences between the electrically and optically measured lifetimes. To acquire experimental lifetime data, $4 \mathrm{H}-\mathrm{SiC}$ substrates with $100-\mu \mathrm{m}$-thick, $1 \times 10^{14} \mathrm{~cm}^{-3}$ to $4.5 \times 10^{14}$ $\mathrm{cm}^{-3}$ doped $\mathrm{N}^{-}$epilayers were prepared; the epilayers had a $2-\mu \mathrm{m}$-thick, $8 \times 10^{18} \mathrm{~cm}^{-3}$ doped $\mathrm{P}^{+}$emitter. A mesa isolation etch was performed to create PiN diodes. One wafer was put through a lifetime enhancement oxidation process, and then both wafers were measured using a commercial $\mu$ PCD system. Front- and backside contacts were formed on both wafers, and the lifetime was subsequently measured using OCVD.

The lifetime measured using the OCVD method was found to depend on the analysis method used, as well as the experimental conditions. Results obtained using a shunt resistance (as in Refs. 8 and 9) were lower than the values measured by $\mu \mathrm{PCD}$, which is an unexpected result due to the OCVD technique's relative independence from surface recombination effects. These erroneous results were due to the damping effect of the external shunt resistance dominating the response for longer carrier lifetimes. Computer-aided analysis of the OCVD transient (as suggested in Ref. 10) yielded results that were higher than the $\mu \mathrm{PCD}$ measured values.

Numerical simulations of the $\mu \mathrm{PCD}$ measurement were used to investigate the transient induced by optical excitation on a structure. Unlike the OCVD measurement, which can be independent of the emitter characteristics for appropriately chosen experimental conditions, ${ }^{10}$ the $\mu$ PCD measurement depends on the total excited carrier concentration present in the epilayer, and can be influenced by the removal of carriers by recombination at the wafer surface as well as the substrate/epilayer interface. The simulations conducted showed that this effect could account for the differences observed between the $\mu \mathrm{PCD}$ and OCVD measurements.

\section{ELECTRICAL AND OPTICAL LIFETIME MEASUREMENTS}

\section{Device Fabrication}

Two commercially grown, $4 \mathrm{H}-\mathrm{SiC}$ substrates cut $4^{\circ}$ towards the (0001) plane with $100-\mu \mathrm{m}$ $1 \times 10^{14} \mathrm{~cm}^{-3}$ to $4.5 \times 10^{14} \mathrm{~cm}^{-3}$ doped $\mathrm{N}^{-}$epitaxial layers were obtained. One of the wafers was subjected to a thermal oxidation treatment to improve the lifetime of the thick epitaxial layer. After the lifetime improvement process, both wafers had a 2 - $\mu$ m-thick, $8 \times 10^{18} \mathrm{~cm}^{-3}$ doped $\mathrm{P}^{+}$anode layer and a $0.5-\mu \mathrm{m}$ very highly doped $\mathrm{P}^{++}$layer grown to form an ohmic contact. The wafers were subjected to a mesa isolation etch to form PiN diodes, followed by a sacrificial oxidation step. A $1-\mu \mathrm{m}$ oxide layer was deposited via plasma-enhanced chemical vapor deposition (PECVD) to passivate the mesa surface, and metal contacts were formed to complete the device structure. The diode active area was $1 \mathrm{~mm} \times 1 \mathrm{~mm}$, or $0.01 \mathrm{~cm}^{2}$.

\section{Optical Lifetime Measurement}

The carrier lifetime was measured using a commercial $\mu \mathrm{PCD}$ lifetime mapping system (Semilab WT-2000) fitted out with a 349-nm third-harmonic $\mathrm{Nd}$ :YLF laser, yielding 2D maps of wafer lifetime with $1 \mathrm{~mm}$ resolution. The measurement system evaluates lifetime by fitting a single exponential function to a portion of the observed decay curve for reflected microwave power versus time, which is an indirect measure of the decay of the optically injected carrier concentration in the semiconductor.

The measurement results for a single $\mu$ PCD line scan from each wafer's secondary flat to the opposing edge are shown in Fig. 1, along with the OCVDmeasured lifetimes corresponding to the same positions. Only the peak lifetimes measured for each die are shown in this plot. The pattern used to form discrete $\mathrm{P}^{+} / \mathrm{N}$ junctions consisted of mesa-isolated scanning the $1 \mathrm{~mm}$ square diode pattern with $0.25 \mathrm{~mm}$ device-to-device spacing with a $1 \mathrm{~mm}$ diameter laser beam results in areas of the wafer being scanned that include all, or part of one of regions not covered by a $\mathrm{P}^{+} / \mathrm{N}$ junction. As these areas have been subjected to etching and plasma processing, the high recombination velocity present in these regions will result in low measured lifetimes; the peaks in the $\mu \mathrm{PCD}$ response correspond to measurements taken when the beam used to excite the semiconductor is directly lined up with the $\mathrm{P}^{+} / \mathrm{N}$ junction regions, and can be related to the OCVD lifetime. The measured lifetime is approximately $4 \mu \mathrm{s}$ in the center of the enhanced wafer, and $1.4 \mu \mathrm{s}$ in the center of the unenhanced wafer. The mean lifetimes and corresponding standard deviations for both the $\mu \mathrm{PCD}$ 
and OCVD techniques on both wafers are listed in Table I.

\section{Electrical Measurement}

Diode lifetime was measured using the OCVD method. ${ }^{11}$ Current pulses were supplied from a HP-314B pulse generator to induce a forward current of approximately $1 \mathrm{~A} / \mathrm{cm}^{2}$ (corresponding to an average injected carrier concentration of about $3 \times 10^{14} \mathrm{~cm}^{-3}$ to $2 \times 10^{15} \mathrm{~cm}^{-3}$ ) in the diode. It was clear from the device current density-voltage $(J-V)$ characteristic that the devices were in the high-level injection regime. Higher current densities (20 A/ $\mathrm{cm}^{2}$, corresponding to an injected carrier concentration between $6 \times 10^{15} \mathrm{~cm}^{-3}$ and $4 \times 10^{16} \mathrm{~cm}^{-3}$ ) did not change the extracted lifetimes. The measurement circuit was similar to that shown in Refs. 8 and 9; however, no parallel resistance was included in the circuit. For longer epilayer lifetimes, any extra shunt resistance can dominate the response of the system, leading to erroneous measurements with low lifetime. The method used to evaluate the minority carrier lifetime was similar to the method employed in Ref. 10. Digitized oscilloscope data were smoothed using the Wolfram Mathematica software package with a moving average function, then the function

$$
\tau_{\mathrm{A}}=2 \frac{k T}{q}\left(\frac{\partial V}{\partial t}\right)^{-1}
$$

was evaluated continuously. The first peak in this function after the cessation of the forward current pulse was taken to be the ambipolar carrier lifetime.

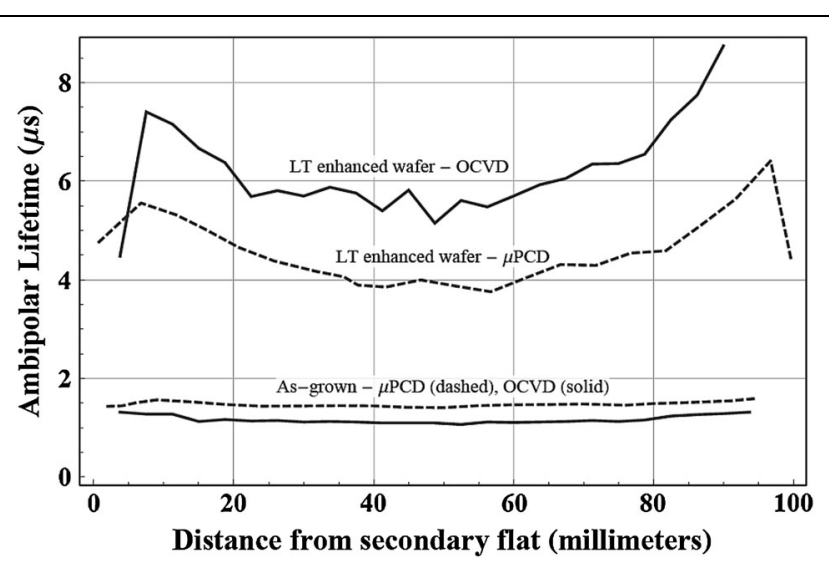

Fig. 1. Ambipolar carrier lifetime, as measured by the $\mu \mathrm{PCD}$ and OCVD methods.
This is illustrated in Fig. 2, which shows the observed voltage decay waveform, along with a line corresponding to the previous equation.

Twenty-five devices across the lateral centerline of each wafer were measured. The measurement results are shown in Fig. 1. The OCVD-measured lifetime is approximately $5.5 \mu \mathrm{s}$ in the center of the enhanced wafer, and $1.1 \mu \mathrm{s}$ in the center of the unenhanced wafer. The difference between the $\mu \mathrm{PCD}$ and OCVD measurements is approximately $2 \mu \mathrm{s}$ in the case of the lifetime-enhanced wafer.

OCVD measurements are expected to yield values for the ambipolar lifetime that are independent of the surface recombination velocity and emitter characteristics: $4 \mathrm{H}-\mathrm{SiC}$ diodes are known to have weak emitters due to the low ionization of acceptors at room temperature, which can lead to significant injection of minority carriers into the emitter region, and their subsequent recombination at the reduced lifetime present in the emitter. While this effect alone would prevent accurate measurement of lifetimes, the loss of carriers due to emitter recombination can be balanced either by diffusion of carriers from the drift to the junction, ${ }^{10,12}$ or by the formation of a potential barrier due to rapid recombination of carriers at the junction interface due to the presence of a thin damaged region. ${ }^{11}$ $\mu \mathrm{PCD}$, on the other hand, can be influenced by surface recombination if carriers diffuse from the emitter/drift boundary to the surface: as the entire carrier density is measured by the $\mu \mathrm{PCD}$ technique, any loss of carriers due to the enhanced recombination rate in the emitter will result in a decrease in the measured reflected radiofrequency (RF) power. This effect was explored through numerical simulations.

\section{Numerical Simulation of Photoexcited Carrier Decay}

The Synopsys Sentaurus commercial semiconductor simulation package was used to simulate the transient characteristics of the $\mu \mathrm{PCD}$ measurement. The input structure consisted of a $100-\mu \mathrm{m}$-thick, $2 \times 10^{14} \mathrm{~cm}^{-3}$ doped N-type epilayer with a $8 \times$ $10^{18} \mathrm{~cm}^{-3}$ doped $\mathrm{P}^{+}$emitter layer. Incomplete ionization of aluminum was modeled in the emitter, resulting in an ionized concentration of $5.6 \times$ $10^{17} \mathrm{~cm}^{-3}$ at room temperature. Doping-dependent lifetime was modeled as in Ref. 13, and the software's default parameters for $4 \mathrm{H}-\mathrm{SiC}$ Auger recombination were used. The top surface of the

Table I. Mean and standard deviation (SD) for lifetime values shown in Fig. 1

\begin{tabular}{|c|c|c|c|c|}
\hline Wafer Type & $\mu$ PCD Mean $\tau_{\mathrm{A}}(\mu \mathrm{s})$ & $\mu \operatorname{PCD}$ SD $\tau_{\mathbf{A}}(\mu \mathbf{s})$ & OCVD Mean $\tau_{\mathrm{A}}(\mu \mathrm{s})$ & OCVD SD $\tau_{\mathbf{A}}(\mu \mathrm{s})$ \\
\hline Lifetime enhanced & 4.57 & 0.69 & 6.21 & 0.92 \\
\hline As-grown & 1.47 & 0.50 & 1.16 & 0.77 \\
\hline
\end{tabular}




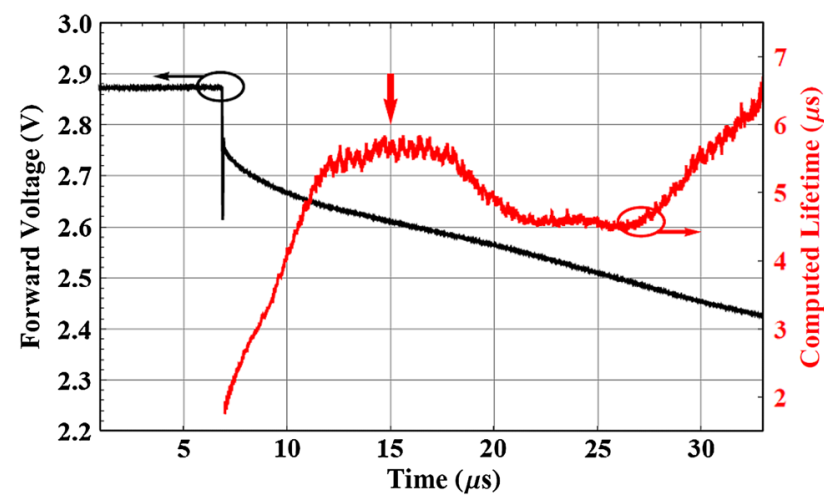

Fig. 2. OCVD transient voltage response and computed lifetime from $\mathrm{PiN}$ diode on lifetime-enhanced $4 \mathrm{H}-\mathrm{SiC}$ wafer. The ambipolar lifetime of $5.6 \mu \mathrm{s}$ is indicated by an arrow.

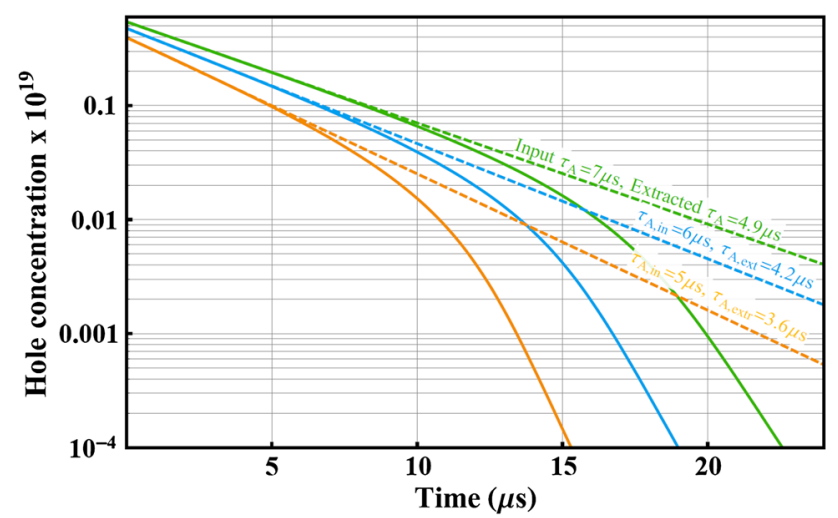

Fig. 3. Simulated photoconductivity decay transient, and exponential fit to high-level injection portion of transient. The vertical axis indicates the integrated hole concentration throughout the simulated volume. The input lifetimes and the extracted exponential decay time constants are labeled on the right.

emitter was described by a surface recombination velocity (SRV) of $1 \times 10^{5} \mathrm{~cm} / \mathrm{s}$, which is a reasonable value expected for a thermally oxidized surface, ${ }^{14}$ and the substrate interface of the epilayer had a SRV of $10 \mathrm{~cm} / \mathrm{s} .{ }^{15}$ The lifetime of the epilayer was also a parameter of the simulation. A contact was assigned to the bottom surface of the epilayer to apply a reference potential for the simulation, but other than that no electrical contacts were included.

An optical generation source incident upon the top of the epilayer with $15 \mathrm{~ns}$ duration was simulated to excite the epilayer into the $p_{n}=4 \times 10^{16}$ $\mathrm{cm}^{-3}$ range near the top surface. An absorption coefficient of $272 \mathrm{~cm}^{-1}$ was included in the simulation, which corresponds to the wavelength of $349 \mathrm{~nm}$ used for the physical $\mu$ PCD lifetime measurements. ${ }^{16}$

Assuming that $\tau_{n}=5 \tau_{p}$ (to highlight the transition from high- to low-level injection) and that the ambipolar lifetime is $\tau_{\mathrm{A}}=\tau_{n}+\tau_{p}$, ambipolar lifetimes from $5 \mu \mathrm{s}$ to $7 \mu \mathrm{s}$ (approximately the range of lifetimes that were observed using OCVD) were simulated using the conditions described above. The integrated carrier concentration in the drift region was plotted versus time, and then a single exponential function was fit to the portion of the decay corresponding to high-level injection conditions. The resulting decay curves and their fit exponential functions are shown in Fig. 3.

The time constant of the exponential fit function is less in each case than the input ambipolar lifetime. The reduction in the lifetime measured from the transient simulation is the result of surface recombination occurring at the anode interface: due to the limited ionization of acceptors at room temperature in $4 \mathrm{H}-\mathrm{SiC}$, in the high-level injection condition, carriers can diffuse through the anode epilayer and recombine at the wafer surface. The approximate $2 \mu$ s difference between the input and calculated lifetimes shown in the simulation is close to the difference between the OCVD and $\mu$ PCD lifetimes measured experimentally. It should be noted that these observed lifetimes are higher than expected for an unpassivated surface without a $\mathrm{P}^{+} / \mathrm{N}$ junction: simulated structures lacking a junction with $5 \mu \mathrm{s}$ and $7 \mu \mathrm{s}$ input ambipolar lifetime and SRV of $1 \times 10^{5} \mathrm{~cm} / \mathrm{s}$ yield extracted lifetimes of only $3.2 \mu \mathrm{s}$ and $3.9 \mu \mathrm{s}$, lower than the values of $3.6 \mu \mathrm{s}$ and $4.9 \mu \mathrm{s}$ predicted by the simulation with a $\mathrm{P}^{+} / \mathrm{N}$ junction.

\section{CONCLUSIONS}

$\mu \mathrm{PCD}$ and OCVD measurements made on $\mathrm{P}^{+} / \mathrm{N}$ diodes fabricated on lifetime-enhanced $4 \mathrm{H}-\mathrm{SiC}$ epilayers yielded lifetime measurements that agreed reasonably well with one another. A discrepancy between the optical and electrical measurements present at higher lifetimes was explained by the removal of carriers in the optical measurement by surface recombination. Due to the simplicity of the numerical simulation models used, it is likely that the discrepancy between OCVD and $\mu$ PCD measurements for this type of thick epilayer and high ambipolar lifetime can be explained by basic semiconductor physics, without the need to consider the complex nature of defect levels leading to lifetime reduction in the material. For lifetime-enhanced thick $\mathrm{SiC}$ epilayers with $\mathrm{P}^{+} / \mathrm{N}$ junctions present, $\mu \mathrm{PCD}$ measurements can provide a good estimate of the minority carrier lifetime for electrical device simulation; however, OCVD measurements conducted using a proper test circuit likely will provide a more accurate estimate due to their independence from surface conditions.

\section{REFERENCES}

1. A. Agarwal, Q. Zhang, R. Callahan, A.B.C. Capell, M. O'Loughlin, J. Palmour, V. Temple, R. Stahlbush, J. Caldwell, H. O’Brien, and C. Scozzie, Mater. Sci. Forum 645-648, 1017 (2010).

2. Q. Zhang, A. Agarwal, C. Capell, L. Cheng, M. O'Loughlin, J.P.A. Burk, S. Rumyantsev, T. Saxena, M. Levenshtein, 
H.O.A. Ogunniyi, and C. Scozzie, Mater. Sci. Forum 717720,1151 (2011).

3. T. Kimoto, Y. Nanen, T. Hayashi, and J. Suda, Appl. Phys. Express 3, 121201 (2010).

4. P.B. Klein, Mater. Sci. Forum 717-720, 279 (2012).

5. P.B. Klein, J. Appl. Phys. 103, 033702 (2008).

6. D.K. Schroder, Semiconductor Material and Device Characterization, 2nd ed. (New York: Wiley Interscience, 1998).

7. S.A. Reshanov and G. Pensl, Mater. Sci. Forum 483-485, 417 (2005)

8. P.A. Losee, C. Li, R.J. Kumar, T.P. Chow, I.B. Bhat, and R.J. Gutmann, Int. J. High Speed Electron. Syst. 17, 43 (2007).

9. P.A. Ivanov, M.E. Levenshtein, K.G. Irvine, O. Kordina, J.W. Palmour, S.L. Rumyantsev, and R. Singh, Int. J. High Speed Electron. Syst. 35, 1382 (1999).
10. S. Bellone, L.F. Albanese, and G. Licciardo, IEEE Trans. Electron Dev. 56, 2963 (2009).

11. M.E. Levinshtein, T.T. Mnatsakanov, P. Ivanov, J.W. Palmour, S.L. Rumyantsev, R. Singh, and S.N. Yurkov, IEEE Trans. Electron Dev. 48, 1703 (2001).

12. H. Schlangenotto and W. Gerlach, Solid-State Electron. 12, 267 (1969).

13. M. Ru, IEEE Trans. Electron Dev. 41, 1040 (1994).

14. A. Galeckas, J. Linnros, M. Frischholz, and V. Grivickas, Appl. Phys. Lett. 79, 365 (2001).

15. P.B. Klein, R. Myers-Ward, K.-K. Lew, B.L. VanMil, C.R. Eddy Jr., D.K. Gaskill, A. Shrivastava, and T.S. Sudarshan, J. Appl. Phys. 108, 033713 (2010).

16. S.G. Sridhara, R.P. Devaty, and W.J. Choke, J. Appl. Phys. 84, 2963 (1998). 